\title{
Wall turbulence closure based on classical similarity laws and the attached eddy hypothesis
}

\author{
A. E. Perry, I. Marusic, and J. D. Li ${ }^{\text {a) }}$ \\ Department of Mechanical and Manufacturing Engineering, University of Melbourne, Parkville, \\ Victoria 3052, Australia
}

(Received 17 May 1993; accepted 8 October 1993)

\begin{abstract}
A new look at the closure problem of turbulent boundary layers is taken here using recently derived analytical expressions for the shear stress distributions. These expressions are based on the logarithmic law of the wall and law of the wake formulation of Coles [J. Fluid Mech. 1, 191 (1956)] with the mean continuity and the mean momentum differential and integral equations. The concept of equilibrium layers of Clauser [Adv. Mech. 4, 1 (1956)] is extended and using similar ideas as Rotta [Prog. Aeronaut. Sci. 2, 1 (1962)] for self-similarity, a closure scheme is proposed for layers developing in arbitrary pressure gradients for the case where the streamwise derivative of the Coles wake factor is not too large. For a given flow case, this Coles wake condition can be tested with internal consistency checks. The mathematical framework is most suitable for incorporating Townsend's attached eddy hypothesis as recently developed by Perry, Li, and Marušić [Philos. Trans. R. Soc. London Scr. A 336, 67 (1991)] for closurc. This gives an opportunity to incorporate coherent structure concepts into closure schemes. Possible ways of handling the difficult case where the streamwise derivative of the Coles wake factor is significant are discussed.
\end{abstract}

\section{INTRODUCTION}

In this work, a new look is taken at the closure problem for turbulent boundary layers in the light of new and more complete analytical expressions recently derived for the shear stress profiles.

One motivation for this work was to incorporate the attached eddy hypothesis of Townsend ${ }^{1}$ as further developed by Perry, Henbest, and Chong ${ }^{2}$ into a closure scheme. Some initial steps towards this are given by Perry, Li, and Marušić. ${ }^{3}$ This represented one of the first attempts to incorporate coherent structure concepts into closure schemes for wall turbulence and avoids the use of local exchange coefficients. This has prompted the following questions for this work:

(1) What sort of mathematical framework would be needed to house such a scheme? Closure schemes such as the attached eddy hypothesis by Perry et $a l^{3}$ use convolution integrals and so differential field methods are clearly inappropriate. Hence an integral scheme is the obvious choice.

(2) What high quality information and what reliable assumptions can we use to feed into this framework so as to minimize arbitrary assumptions for closure?

(3) At what point in the framework does closure naturally enter and what are the most natural nondimensional parameters to use?

For (2) above, the following have been chosen:

(a) The logarithmic law of the wall and the law of the wake.

\footnotetext{
a) Present address: Department of Mechanical Engineering, University of Sydney, N.S.W. 2006, Australia.
}

(b) The mean momentum differential and integral equations.

(c) The mean continuity equation.

(d) The assumption that streamwise derivatives of the normal Reynolds stresses are negligible.

(e) The assumption that close to precise twodimensional mean flow is possible.

(f) The belief that if theoretical conditions can be found from the mean momentum and continuity equations which are consistent with self-similarity in both the velocity defect and shear stress profiles, then when these conditions are applied, such selfsimilarity will indeed occur (e.g. see Rotta ${ }^{4}$ ).

Assumption (a) is consistent with the classical approach to wall turbulence using the similarity schemes of Millikan, ${ }^{5}$ Clauser, ${ }^{6,7}$ and Rotta. ${ }^{4}$

Using assumptions (a) to (e) above, the shear stress relationships have been derived. Further, with the aid of (f) above, evolution equations for the streamwise development of equilibrium layers have been derived. This is extended to quasiequilibrium layer development where the Coles wake factor is permitted to vary slowly with streamwise distance. The required relationships for closure and the most appropriate nondimensional parameters to use are derived. The form of these closure relationships would need to be obtained from a series of systematic "once and for all" experiments. It might also be possible to use the attached eddy hypothesis in conjunction with this. An example of this is illustrated later in the paper.

For the case of Coles wake factor undergoing rapid streamwise increase, the quasiequilibrium assumptions breakdown. Problems with this are left to future work but possible directions are briefly discussed.

Much of this work in deriving the shear stress profile 


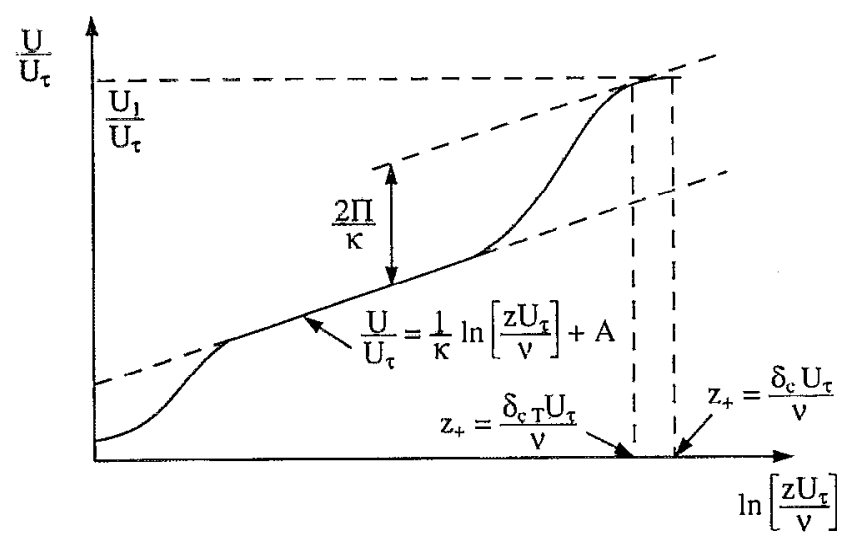

FIG. 1. Details of how various wake parameters are defined.

expressions is based on the work of Coles, ${ }^{8} \mathrm{Li}^{,}{ }^{9}$ Marušić, ${ }^{10}$ Perry and $\mathrm{Li}^{11}$ and Perry. ${ }^{12,13}$ Parts of this paper are based on a more detailed G.A.L.C.I.T. report by Perry. ${ }^{13}$ As far as possible results have been kept in analytical form and here MATHEMATICA was found to be most useful.

\section{SHEAR STRESS PROFILES IN TURBULENT BOUNDARY LAYERS}

We start with the Coles ${ }^{14}$ logarithmic law of the wall, law of the wake formulation. This can be written as

$$
\frac{U}{U_{\tau}}=\frac{1}{\kappa} \ln \left[\frac{z U_{\tau}}{v}\right]+A+\frac{\Pi}{\kappa} W_{c}[\eta, \Pi]
$$

where $U$ is the mean velocity in the streamwise direction (i.e., the $x$ direction), $z$ is the distance normal to the wall, $U_{\tau}$ is the friction velocity given by $U_{\tau}=\left(\tau_{0} / \rho\right)^{1 / 2}$ where $\tau_{0}$ is the wall shear stress and $\rho$ is the fluid density, $v$ is the fluid kinematic viscosity, $\kappa$ is the von Kármán constant (later assumed to be 0.41 ), $A$ is the universal smooth wall constant (later assumed to be 5.1 ), $\Pi$ is the Coles wake factor which generally varies with streamwise distance and $W_{c}$ is the Coles wake function. Traditionally $W_{c}$ is a universal function of $\eta_{T}$ alone where $\eta_{T}=z / \delta_{c T}$ where $\delta_{c T}$ is the traditional Coles thickness which is located at the point of maximum deviation from the semilogarithmic law (see Fig. 1). However we will use the Lewkowicz ${ }^{15}$ formulation, Eq. (2), which ensures that $\partial U / \partial z=0$ at $\eta=1$ where $\eta=z / \delta_{c}$ and $\delta_{c}$ is also shown in Fig. 1, i.e.

$$
W_{c}[\eta, \Pi]=2 \eta^{2}(3-2 \eta)-\frac{1}{\Pi} \eta^{2}(1-\eta)(1-2 \eta) .
$$

An alternative formulation, which also satisfies the above condition, was used by Perry and $\mathrm{Li}^{16,11}$ and Marušic ${ }^{10}$ and is of the form

$$
W_{c}[\eta, \Pi]=1-\cos [\gamma \pi \eta]
$$

where

$$
\gamma=\delta_{c} / \delta_{c T}
$$

and

$$
1+\Pi \gamma \pi \sin [\gamma \pi]=0 .
$$

This trigonometric formulation is unsuitable for low values of $\Pi$ since for $\Pi$ less than about 0.2 , the solution for $\gamma$ follows an unrealistic branch. Also the polynomial formula was found to be slightly more convenient for computing shear stress profiles and mean profile parameters.

In this work the velocity defect formulation will be used throughout. That is

$$
\begin{aligned}
\frac{U_{1}-U}{U_{\tau}} & =f[\eta, \mathrm{II}] \\
& =-\frac{1}{\kappa} \ln \eta+\frac{\Pi}{\kappa} W_{c}[1, \Pi]-\frac{\Pi}{\kappa} W_{c}[\eta, \Pi]
\end{aligned}
$$

where $U_{1}$ is the local free-stream vclocity.

The mean continuity equation is

$$
\frac{\partial U}{\partial x}+\frac{\partial W}{\partial z}=0
$$

where $W$ is the mean velocity component normal to the wall. Note that two-dimensional mean flow is being assumed.

The mean momentum equation is

$$
U \frac{\partial U}{\partial x}+W \frac{\partial U}{\partial z}=-\frac{1}{\rho} \frac{d p}{d x}+\frac{1}{\rho} \frac{\partial \tau}{\partial z}
$$

where $p$ is the free-stream static pressure and $\tau$ is the local shear stress. It should be noted that streamwise gradients of turbulence normal stresses are being neglected. Later the relationship

$$
\frac{\tau}{\rho}=-\overline{u^{\prime} w^{\prime}}+v \frac{\partial U}{\partial z}
$$

will be used. Here $v \partial U / \partial z$ is the viscous contribution and $-\overline{u^{\prime} w^{\prime}}$ is the Reynolds (kinematic) shear stress where $u^{\prime}$ and $w^{\prime}$ are the fluctuating components of velocity in the $x$ and $z$ directions respectively and the overbar denotes a time average. For the region of flow where $z U_{\tau} / v>50$, the viscous contribution is negligible. Perry, ${ }^{12}$ who considered equilibrium layers found that the inclusion of the buffer zone and viscous sublayer in the formulation had negligible effect on the overall momentum balance and shear stress distribution for practical ranges of Reynolds number and it is assumed here that it is quite safe for the purpose of momentum balances and shear stress profilcs to take the logarithmic law profile all the way to the wall.

Substituting (4) and (5) into (6) and making use of relations derived from the logarithmic law of the wall and momentum integral equation we obtain, after much algebra

$\frac{\tau}{\tau_{0}}=f_{1}[\eta, \Pi, S]+f_{2}[\eta, \Pi, S] \delta_{c} \frac{d \Pi}{d x}+f_{3}[\eta, \Pi, S] \frac{\delta_{c}}{U_{1}} \frac{d U_{1}}{d x}$.

Here

$$
S=U_{1} / U_{\tau}=\sqrt{2 / C_{f}^{\prime}}
$$

where $C_{f}^{\prime}$ is the local skin friction coefficient, given by 

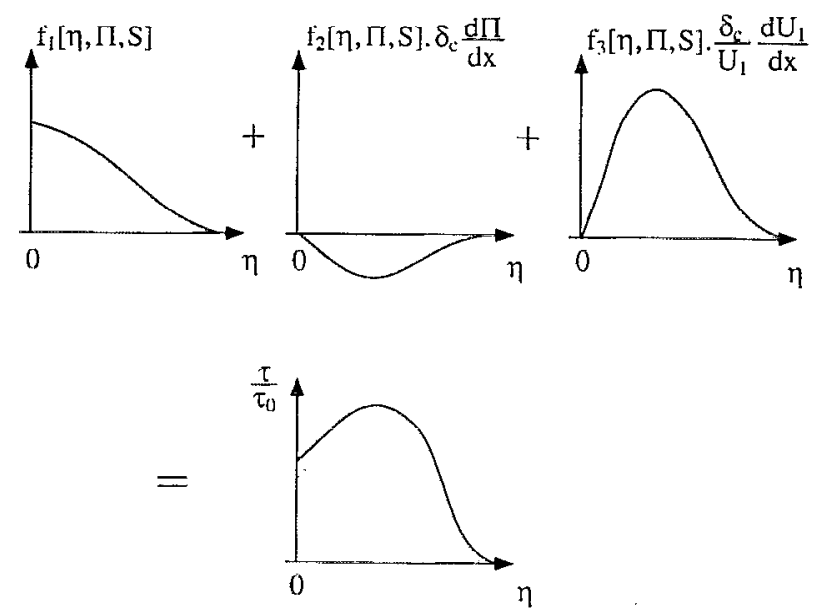

FIG. 2. Components of Eq. (8) for a typical adverse pressure gradient case.

$$
C_{f}^{\prime}=\frac{\tau_{0}}{\frac{1}{2} \rho U_{1}^{2}}
$$

Functions $f_{1}$ and $f_{2}$ and $f_{3}$ are complicated and their detailed forms have been deduced by Perry ${ }^{13}$ and some details are given in the Appendix. Equation (8) is the simplest and most convenient form so far derived and it can be seen that there are three components of stress given and these are shown diagrammatically in Fig. 2 for a typical adverse pressure gradient boundary layer. The first term gives a shear stress distribution which resembles a zero pressure gradient layer. The third term adds a curve with a positive maximum but the second term is negative. A positive $\delta_{c} d \Pi / d x$ causes a reduction in the stress distribution. In the case of favorable pressure gradients to be considered later with numerical examples, the signs of the second and third terms are opposite to that of adverse pressure gradients.

In an equilibrium layer, the second term in (8) is zero and for adverse pressure gradients, the nondimensional shear stress reaches the highest possible maximum value. This is an interesting property of equilibrium boundary layers.

Figure 3 shows some experimental data of Marušić ${ }^{10}$ for a nonequilibrium layer compared with Eq. (8). Here, $\delta_{c} d \Pi / d x,\left(\delta_{c} / U_{1}\right) d U_{1} / d x$, and $S$ were obtained from experiment and fed into ( 8 ) and the agreement can be seen to be satisfactory. Figure 4 shows results which the authors interpolated from the data of East, Sawyer, and $\mathrm{Nash}^{17}$ who carried out a series of experiments on a family of equilibrium layers (i.e. $S \delta_{c} d \mathrm{II} / d x$ was approximately zero-see Sec. V). The interpolation gives the same values of $\Pi$ as for Marušic's data and the results are plotted to the same scale. It can be seen that the effect of $\delta_{c} d \Pi / d x$ is very large in the case of Marušić's data and the maximum nondimensional shear stress is considerably lower than the East et al. data. Thus there is no one to one correspondence between the nondimensional velocity defect profile (characterized by $\Pi$ ) and the nondimensional shear stress

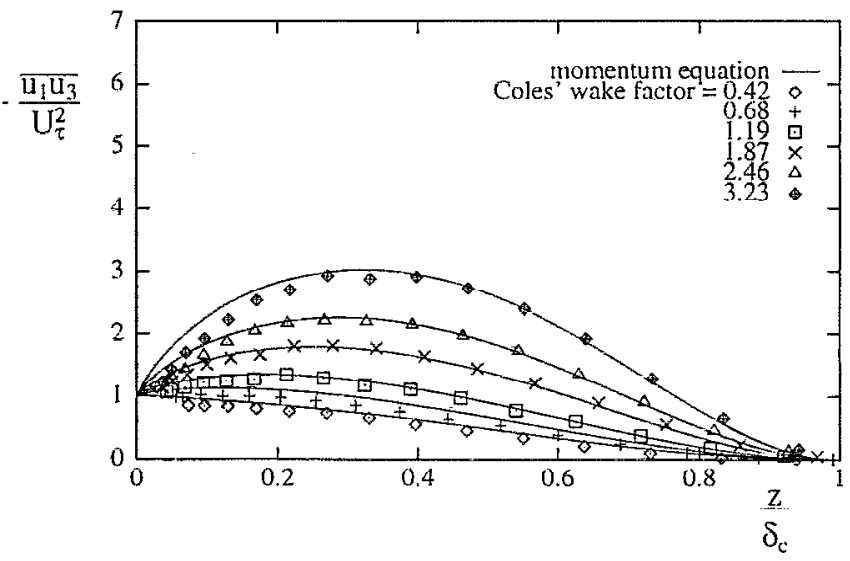

FIG. 3. Nonequilibrium flow data of Marusić (Ref. 12). Solid lines are obtained using Eq. (8).

profiles as is implied in theories which use universal distributions of eddy viscosity or mixing length. From what follows later, such theories would come to grief if $\delta_{c} d \Pi / d x$ effects are significant.

Equation (8) has been rigorously derived and so is an excellent tool for testing experimental data. Perry and $\mathrm{Li}^{11}$ applied (8) to the Reynolds shear stress data of many workers. Departures indicate either a lack of twodimensionality or poor hot-wire techniques, assuming of course that the law of the wall and law of the wake formulation is correct.

\section{EVOLUTION EQUATIONS FOR EQUILIBRIUM BOUNDARY LAYERS}

The equations which govern the streamwise evolution of turbulent boundary layers will be given. Our attention for the moment will be confined to equilibrium layers and hence $\delta_{c} d \Pi / d x=0$. We will use the logarithmic law of the wall, law of the wake and the momentum integral equation. This latter equation is

$$
\frac{d \theta}{d x}+\left(\frac{\delta^{*}}{\theta}+2\right) \frac{\theta}{U_{1}} \frac{d U_{1}}{d x}=\frac{1}{S^{2}}
$$

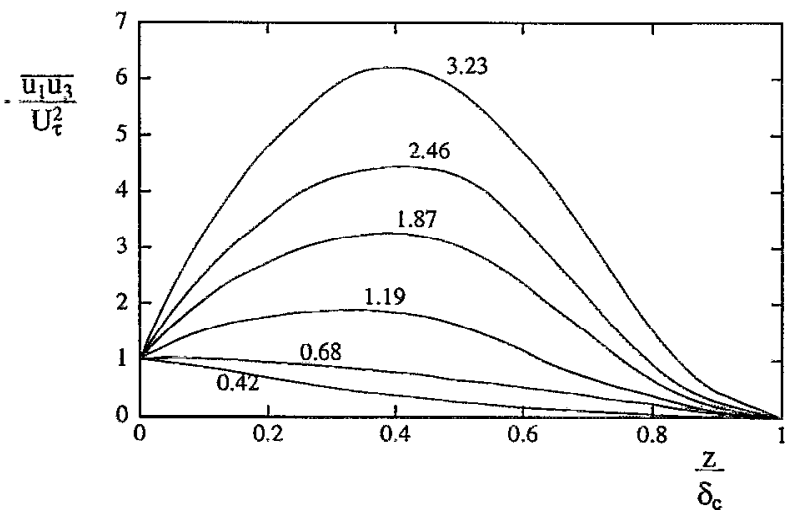

FIG. 4. Authors interpolated data for the same values of $I I$ as shown in Fig. 3 for the approximate equilibrium flows of East et al. (Ref. 17). 
where $\theta$ and $\delta^{*}$ are the momentum and displacement thicknesses respectively and are given by

$$
\begin{aligned}
& \frac{\theta}{\delta_{c}}=\frac{C_{1}}{S}-\frac{C_{2}}{S^{2}}, \\
& \frac{\delta^{*}}{\delta_{c}}=\frac{C_{1}}{S}
\end{aligned}
$$

where

$$
C_{1}[\Pi]=\int_{0}^{1} f[\eta, \Pi] d \eta=\int_{0}^{\infty} \frac{U_{1}-U}{U_{\tau}} d \eta
$$

and

$$
C_{2}[\Pi]=\int_{0}^{1} f^{2}[\eta, \Pi] d \eta=\int_{0}^{\infty}\left(\frac{U_{1}-U}{U_{\tau}}\right)^{2} d \eta .
$$

The analysis which follows makes use of the following definitions and identities:

$$
\frac{\delta_{c}}{U_{1}} \frac{d U_{1}}{d x}=-\frac{\beta}{C_{1} S}
$$

where

$$
\beta=\frac{\delta^{*}}{\tau_{0}} \frac{d p}{d x}
$$

where $\beta$ is the Clauser ${ }^{7}$ pressure gradient parameter.

From the logarithmic law of the wall and law of the wake

$$
\frac{\delta_{c} U_{\tau}}{\nu}=K_{\tau}=E[\Pi] \exp [\kappa S] .
$$

Here $K_{\tau}$ will be referred to as the von Kármán number; $E[\Pi]$ is given by

$$
E[\Pi]=\exp \left[-\kappa\left(A+(\Pi / \kappa) W_{c}[1, \Pi]\right)\right] .
$$

After a considerable amount of algebra, we arrive at two evolution equations which are given as (20) and (21) below.

$$
\begin{aligned}
& S E[\Pi] \exp [\kappa S] \frac{1}{\chi} \frac{d S}{d R_{x}}=R[S, \beta, \Pi], \\
& S^{2} E[\Pi] \exp [\kappa S] \frac{1}{\chi^{2}} \frac{d \chi}{d R_{x}}=-\frac{\beta}{C_{1}[\Pi]},
\end{aligned}
$$

where

$$
\chi=\frac{U_{1}}{U_{0}}=\sqrt{1-C_{p}}
$$

where $C_{p}$ is the local free-stream pressure coefficient. Also

$$
R_{x}=\frac{x U_{0}}{v}
$$

and

$$
R=\frac{2 \beta C_{1} S+C_{1} S-\beta C_{2}}{C_{1}\left\{\kappa S^{2} C_{1}-\kappa S C_{2}+C_{2}\right\}}
$$

where $U_{0}$ is the free-stream velocity at $x=0 ; R_{x}$ is the most practical of Reynolds numbers since it is based on variables explicitly specified by the user. Some of the intermediate equations used in arriving at the above relationships are given in the Appendix [Eqs. (A6) to (A10)].

Equation (20) is derived from the momentum integral equation and the logarithmic law of the wall and the law of the wake. Equation (21) comes purely from the logarithmic law of the wall and the law of the wake.

Equations (20) and (21) are a pair of coupled equations which have been derived for equilibrium layers where $\Pi$ is invariant with streamwise Reynolds number $R_{x}$. One must keep in mind that $E, C_{1}$, and $C_{2}$ are all functions of II alone and so are also invariant with $R_{x}$. A third equation is needed for closure. From an extension of the work of Coles $^{8}$ and Clauser ${ }^{7}$ we need to know the functional form

$$
\beta=\beta[\Pi, S] .
$$

For further clues regarding (25) consider the following. For a true equilibrium layer we should have self-similarity in both velocity defect and the shear stress distributions but according to Rotta ${ }^{4}$ precise self-similarity is not possible on a smooth surface except for equilibrium sink flows. This will be considered later. However, conditions for an approximate self-similarity can be obtained with the aid of (8). Since $\delta_{c} d \Pi / d x=0$ then (8) with (16) gives

$$
\frac{\tau}{\tau_{0}}=f_{1}[\eta, \Pi, S]-f_{3}[\eta, \Pi, S] \frac{\beta}{C_{1} S} .
$$

Although it is not possible to collapse $\tau / \tau_{0}$ for all $\eta$ at a fixed II, let us seek conditions necessary for forcing the profiles to match at $\eta=m$ where $m$ will be chosen to give reasonable collapse for most of the profile. In other words

$$
\left(\frac{\tau}{\tau_{0}}\right)_{\eta=m}=f_{1}[m, \Pi, S]-f_{3}[m, \Pi, S] \frac{\beta}{C_{1} S}=\mathscr{L}[\Pi, m]
$$

where in (27) $\beta$ and $S$ will be varied for fixed $\Pi$ in such a way as to produce a fixed $\mathscr{L}$. It has been found ${ }^{13}$ that $m=0.4$ appears to be close to an optimum choice for close to precise self-similarity for a wide range of $\Pi$ and for all $S$. It turns out that $f_{1}[m, \Pi, S]$ is a ratio of two polynomials in $S$ of the same order and as $S \rightarrow \infty, f_{1}$ approaches a constant. Also $f_{3}[m, \Pi, S] / C_{1} S$ has similar properties to $f_{1}$ and as $S \rightarrow \infty, f_{3} / C_{1} S$ approaches a constant. Hence for $S \rightarrow \infty$, $\beta \rightarrow$ const for fixed $\mathscr{S}$.

It has been shown by Perry ${ }^{13}$ that for the cases considered here the general behavior of $S \rightarrow \infty$ as $R_{x} \rightarrow \infty$ applies. From (27) it can be seen that $\beta$ must approach an asymptotic value $\beta_{g}[\Pi]$ which is a function of $\Pi$ alone. In order to obtain closure, it is necessary to know the function $\beta_{g}[\Pi]$. East $e t a l .{ }^{17}$ carried out a series of experiments on a family of equilibrium layers and assumed that $\beta$ was a function only of II and data seem to fit a relationship proposed by Green et al. ${ }^{18}$ which is

$$
\beta_{g}[\Pi]=\left(0.024\left(C_{2}[\Pi] / C_{1}[\Pi]\right)^{2}-1\right) / 0.8 \text {. }
$$

This is shown later in Fig. 19 and it should be noted that for $I I=0, \beta_{g} \approx-1 / 2$ which is consistent with the work of Jones and Launder ${ }^{19}$ for equilibrium sink flow at infinite 
Reynolds numbers. Here the suffix $g$ denotes Green et al. ${ }^{18}$ Other similar curve fits for $\beta$ versus $\Pi$, showing little difference to (28), have been proposed by Mellor and Gibson, ${ }^{20}$ see White. ${ }^{21}$

Although East et al.'s experiments were not carried out for $S \rightarrow \infty$, for the purpose of this preliminary investigation it will be assumed that $\beta_{g}$ corresponds with the asymptotic value of $\beta$ for fixed $\mathscr{L}$. This is a temporary assumption just to develop the mathematics and numerical schemes. When and if a more accurate version of (28) is found it will be replaced.

Let $\beta$ variations for a fixed $\mathscr{L}$ and a given asymptotic value of $\beta_{g}$ be denoted by

$$
\beta=\beta_{\ell g}[\Pi, S]
$$

and

$$
\beta_{g}=\beta_{l g}[\Pi, \infty]
$$

and it is understood that $m=0.4$.

Applying Eq. (27) for finite $S$ and infinite $S$ we obtain

$$
\begin{aligned}
\beta_{\ell g}[\Pi, S]= & \frac{C_{1}[\Pi] S}{f_{3}[0.4, \Pi, S]}\left\{f_{1}[0.4, \Pi, S]-f_{1}[0.4, \Pi, \infty]\right. \\
& \left.-\lim _{S \rightarrow \infty}\left\{\frac{f_{3}[0.4, \Pi, S]}{C_{1}[\Pi] S}\right\}_{g}[\Pi]\right\} .
\end{aligned}
$$

Equation (31) gives a known analytical function for (29).

Clauser ${ }^{6,7}$ proposed that an equilibrium layer is produced if $\beta$ is fixed for a fixed П. From work here it is seen that this is true only asymptotically. As a rough guide to the results of (31), for $\Pi=0.1, \beta$ increases by about $25 \%$ over the entire range of $S$ and for $\Pi=10, \beta$ decreases by about $20 \%$ over the range. See Perry ${ }^{13}$ for details. Other theories have been reviewed by Perry ${ }^{13}$ and it was shown that their performance for producing self-similar shear stresses was poor.

\section{THE QUASIEQUILIBRIUM HYPOTHESIS}

Let us now assume that these equations can be applied to nonequilibrium boundary layers provided that $\delta_{c} d \Pi / d x$ is sufficiently small and that local values of $\Pi$ for a given $R_{x}$ can be used in (20) and (21). Such layers will be referred to as quasiequilibrium layers. One measure for sufficient smallness of $\delta_{c} d \Pi / d x$ can be derived from the shear stress equation (8) i.e.,

$$
\frac{f_{2}[m, \Pi, S] \delta_{c} d \Pi / d x}{f_{1}[m, \Pi, S]+f_{3}[m, \Pi, S]\left(\delta_{d} / U_{1}\right)\left(d U_{1} / d x\right)} \ll 1 \text {. }
$$

Given then condition (32), Eqs. (20) and (21) will be applied to some interesting nonequilibrium flow cases. So as to keep the study as analytical as possible, streamwise pressure distributions will be chosen which can be characterized by one single parameter. Such flows are sink and source flows.

\section{A. Favorable pressure gradients}

Let us consider sink flows. These are shown diagrammatically in Fig. 5 and if boundary layer displacement effects are neglected, continuity gives

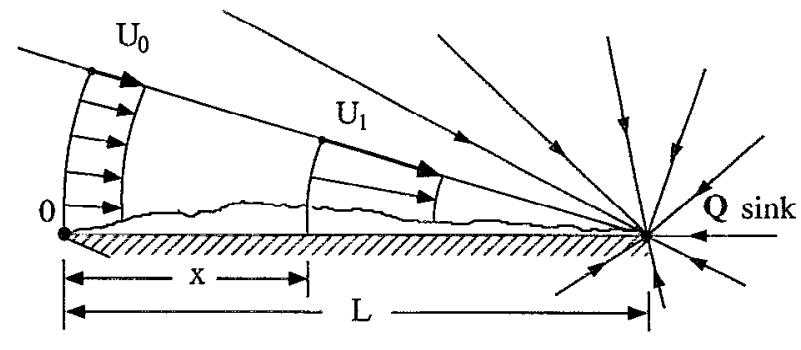

FIG. 5. Sink flow.

$$
\frac{U_{1}}{U_{0}}=\chi=\frac{1}{1-K R_{x}},
$$

where

$$
K=\frac{v}{L U_{0}}
$$

and $K$ is often referred to as the acceleration parameter. In fact $K$ is related to the sink strength, thus

$$
K=\frac{2 \pi v}{Q} .
$$

It is easy to further show that

$$
\frac{1}{\chi^{2}} \frac{d \chi}{d R_{x}}=K \text {. }
$$

Equation (21) can be written as

$$
K C_{1}[\mathrm{II}] S^{2} E[\mathrm{II}] \exp [\kappa S]-\beta_{\ell_{8}}[S, \mathrm{II}]=0,
$$

i.e.,

$$
F[K, \Pi, S]=0 .
$$

Now $\beta_{\ell g}$ is known analytically from (31) and Eq. (36) can be solved for $\Pi$ at fixed $K$ and $S$ numerically. This gives a typical plot shown in Fig. 6.

Equation (33) and the results of (36) are substituted into $(20)$ to give

$$
R_{x}=\frac{1}{K}\left\{1-\exp \left[K \int_{S_{0}}^{S} H[S] d s\right]\right\}
$$

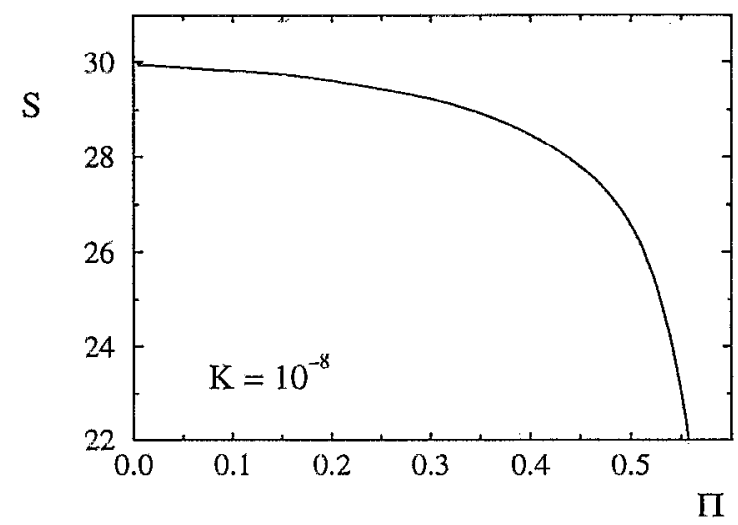

FIG. 6. II vs $S$ for sink flow, from solution of Eq. (36). 


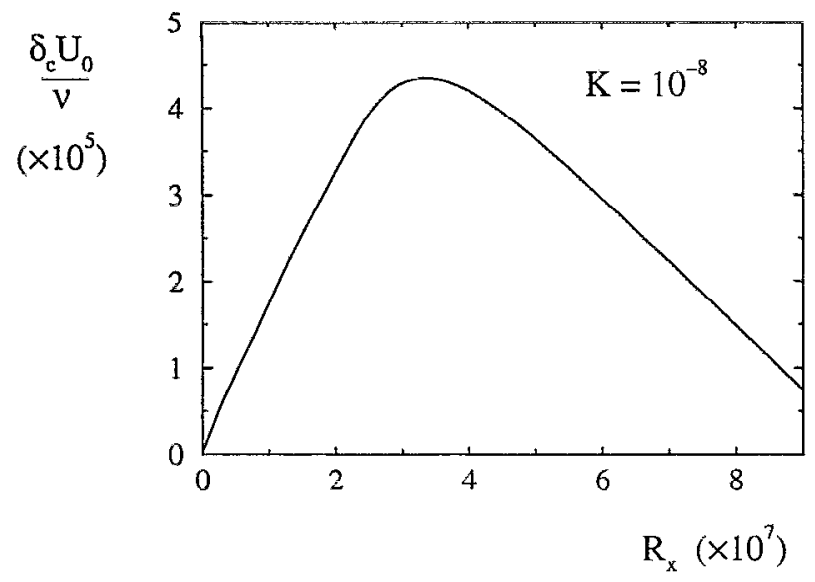

FIG. 7. Sink flow boundary layer growth with $R_{x}$.

where

$$
H[S]=\frac{S E[\Pi[S]] \exp [\kappa S]}{R\left[S, \beta_{\ell g}[\Pi[S], S], \Pi[S]\right]} .
$$

Here $S_{0}$ is the initial value of $S$ and has been chosen such that $K_{\tau}$ in (18) is of order 100 . It is expected that this would be appropriate for a boundary layer which has been freshly tripped. If however, one is interested only in values of $R_{x}$ which are high, the results should be insensitive to the precise value of $S_{0}$ chosen. Once $R_{x}$ versus $S$ has been found, all other profile parameters can be found.

Figure 7 shows a case where $K=10^{-8}$ and one can see that the layer initially thickens and then thins linearly to zero thickness when $R_{x}=10^{8}$. From Fig. 8 it appears that $S$ asymptotes to a constant value invariant with $R_{x}$ and thus satisfies the Rotta ${ }^{4}$ conditions for precise equilibrium flow. Figure 9 shows shear stress profiles given by Eq. (8). Since the quasiequilibrium results give $\Pi$ versus $R_{x}$, the quantity $\delta_{c} d \Pi / d x$ can be calculated and the shear stress profiles can be calculated with the $\delta_{c} d \Pi / d x$ effect included and with it excluded. If it is included, the shear stress profiles are higher since $\delta_{c} d \Pi / d x$ is negative. Both sets of profiles have been calculated and the discrepancy can be

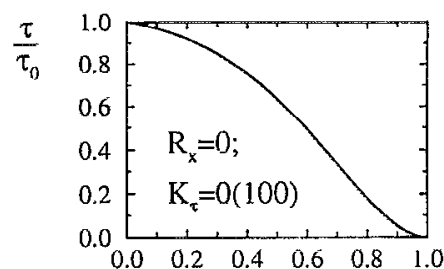

(a)

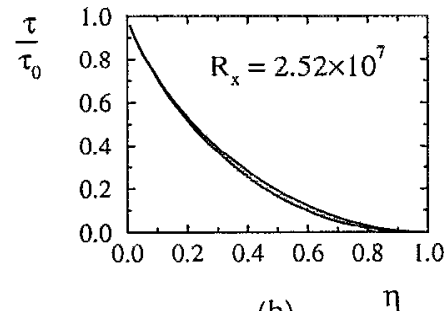

(b)

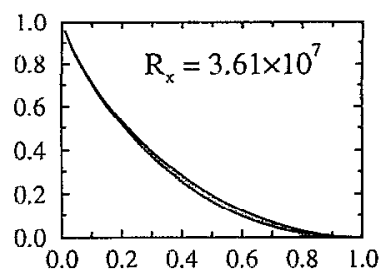

(c)

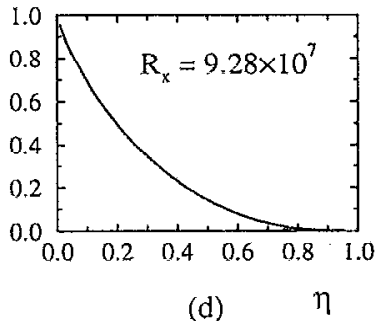

(d)

FIG. 9. Sink flow shear stress profiles given by Eq. (8) with and without the inclusion of the $\delta_{c} d \Pi / d x$ term. In all cases, $K=10^{-8}$; (a) $\Pi \approx 0.55$, (b) $\mathrm{II} \approx 0.15$, (c) $\Pi \approx 0.06$, and (d) $\Pi \approx 0.02$.

seen in the profiles for the region where the boundary layer is growing. Thus the method has a built-in "alarm" which warns the user if the assumption of quasiequilibrium is breaking down.

An additional interesting point about Eqs. (20) to (24) is that if $d S / d R_{x}=0$, then as $S \rightarrow \infty, \beta_{\ell g} \rightarrow-1 / 2$ (see Perry ${ }^{13}$ ).

\section{B. Zero pressure gradient flows}

Here we put $K=0$ and with the aid of (33) and (35), (21) gives

$$
\beta_{\ell g}[S, \Pi]=0
$$

and so

$$
\Pi=\Pi[S]
$$

can be found and (20) gives

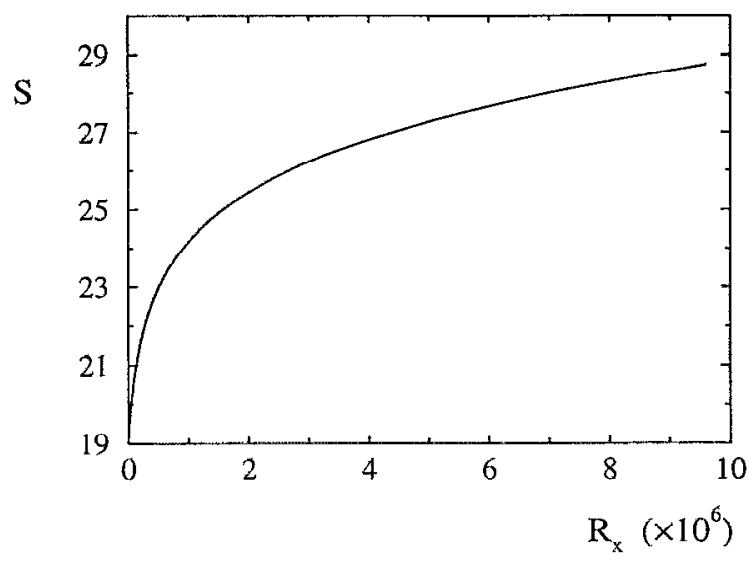

FIG. 10. $S$ vs $R_{x}$ for zero pressure gradient flow $(K=0)$.

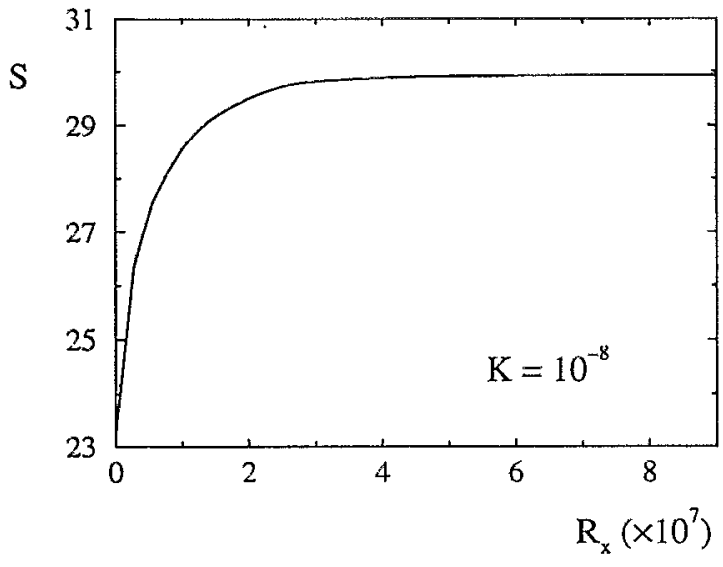

FIG. 8. $S\left(\left(2 / C_{f}^{\prime}\right)^{1 / 2}\right)$ vs $R_{x}$ for sink flow. 


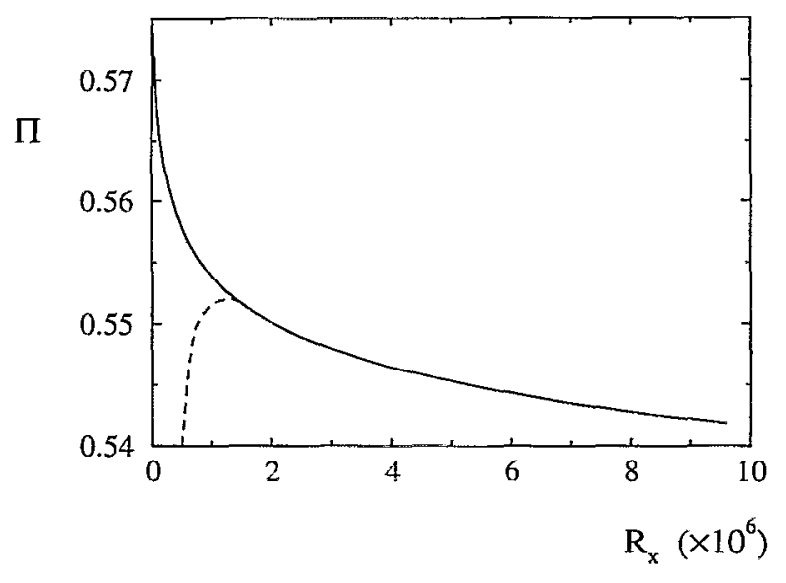

FIG. 11. $\Pi$ vs $R_{x}$ for zero pressure gradient flow ( $K=0$ ). Broken line indicates approximate trend with inclusion of "low Reynolds number effect."

$$
R_{x}=\int_{S_{0}}^{S} \frac{S E[\Pi[S]] \exp [\kappa S]}{R[S, 0, \Pi[S]]} d S .
$$

With this formulation one can see that a zero pressure gradient layer is not an equilibrium layer at least for finite $S$ since II varies with $R_{x}$, albeit slowly. Figures 10 and 11 show the evolution of $S$ versus $R_{x}$ and $\Pi$ versus $R_{x}$. Coles ${ }^{22}$ has pointed out that for $R_{\theta}$ less than about 2500 , II drops to zero for decreasing $R_{\theta}$. This low Reynolds number effect corresponds approximately with the broken line in Fig. 11. This effect has not been incorporated into the analysis. This should have a negligible effect on flow at high $R_{x}$.

Figure 12 shows the calculated shear stress profiles with and without the effect of $\delta_{c} d \Pi / d x$ included. It can be seen that $\delta_{c} d \Pi / / d x$ has no effect and although the shear stress profiles do vary with $R_{x}$, they are in true quasiequilibrium. If we assume that $\Pi=$ const and put $\beta=0$, Eq. (40) will yield the classical von Kármán law of skin friction, see Perry. ${ }^{13}$

\section{Adverse pressure gradient flows}

For adverse pressure gradients being produced by a source, the analysis is the same as for favorable pressure

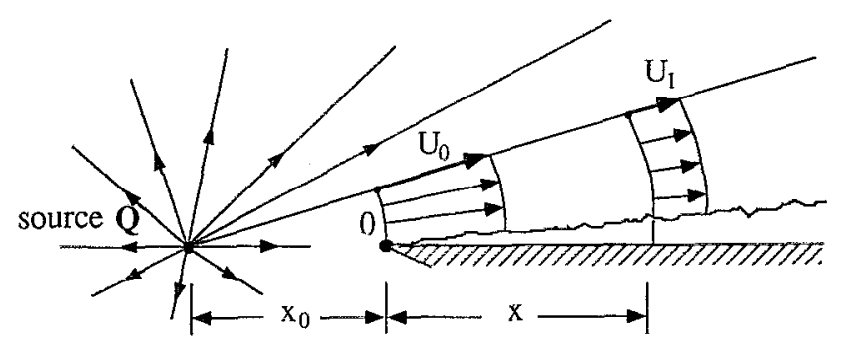

FIG. 13. Source flow.

gradients except $K=-\left(x_{0} U_{0} / v\right)^{-1}$ where $x_{0}$ and $U_{0}$ are defined in Fig. 13. Figure 14 shows the calculated shear stress profiles for $K=-7.5 \times 10^{-8}$ with and without the effect of $\delta_{c} d \Pi / d x$. The effect of $\delta_{c} d \Pi / d x$ does not appear to be particularly strong and so perhaps this also represents a case of quasiequilibrium flow.

\section{NON-QUASIEQUILIBRIUM FLOW}

It is quite obvious that for more general flow cases evolution equations need to be derived with an account made for the effects of $d \Pi / d x$. Equation (8) shows that $f_{2}[\eta, \Pi, S] \rightarrow \infty$ as $S \rightarrow \infty$, i.e. it has a similar behavior as $f_{3}[\eta, \Pi, S]$ treated in Sec. III. Since we expect $\tau / \tau_{0}$ to remain finite as $S \rightarrow \infty$, then a more appropriate parameter for derivatives of $\Pi$ is $S \delta_{c} d \Pi / d x$ ( $=\zeta$ say) and its coefficient in Eq. (8) will be $f_{2}[\eta, \Pi, S] / S$ which is finite as $S \rightarrow \infty$. Thus we will have evolution equations involving $d \Pi / d x$ and shear stress profile equations involving $\zeta$. Furthermore, a simple relationship between the asymptotic value of $\beta$ (i.e., $\beta_{a}$ say) and $\Pi$ will no longer suffice for obtaining closure. For quasiequilibrium $\beta_{a}=\beta_{g}$ with $\beta_{g}$ given by a curve fit like Eq. (28). However, this would need to be replaced by some sort of multidimensional fit and as a first attempt to consider a broader class of layers we could try

$$
\beta_{a}=\beta_{a}[\Pi, \zeta]
$$

and that in general

$$
\beta=\beta[S, \Pi, \zeta] \text {. }
$$

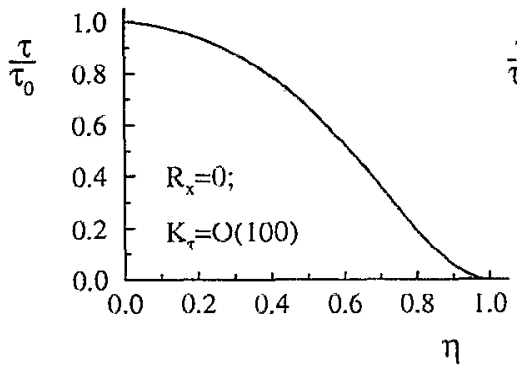

(a)

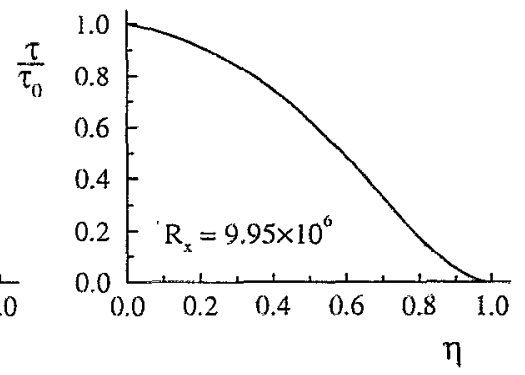

(b)

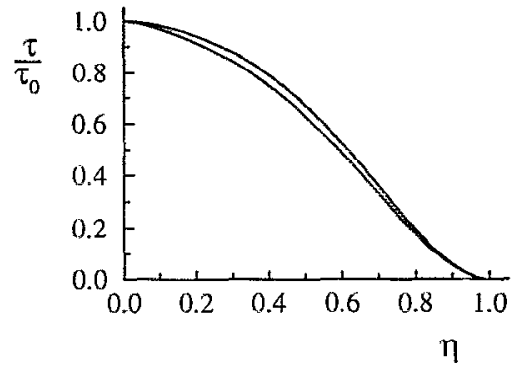

(c)

FIG. 12. Zero pressure gradient flow $(K=0)$ shear stress profiles given by Eq. (8) with and without the inclusion of the $\delta_{c} d \Pi / d x$ term. Note that (a) and (b) contain two data sets each-no difference is visible; (c) is a combined plot of the data sets from (a) and (b) to illustrate the Reynolds number dependence. 


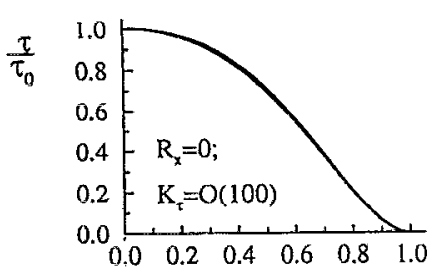

(a)

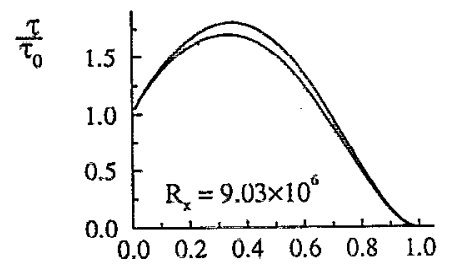

(b)

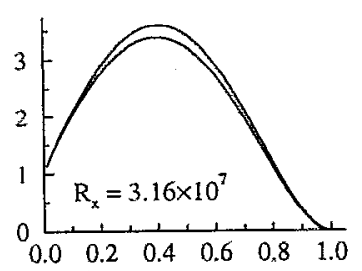

(c)

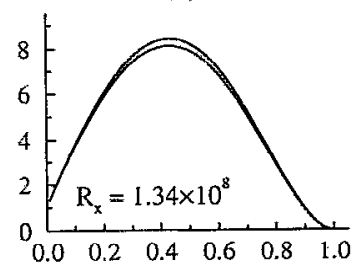

(d)

n
FIG. 14. Source flow shear stress profiles given by Eq. (8) with and without the inclusion of the $\delta_{c} d \Pi / d x$ term. In all cases, $K=-7.5 \times 10^{-8}$ : (a) $\Pi \approx 0.55$, (b) $\Pi \approx 1.0$, (c) $\Pi \approx 2.0$, and (d) $\mathrm{II} \approx 3.5$.

It is conjectured that an analysis similar to that for equilibrium flow can be carried out which will yield (42) by forcing shear stress profiles to be invariant with $S$. In quasiequilibrium flow it was assumed that if $\Pi$ is fixed so also is the shear stress distribution (i.e., $S$ is not involved). By analogy, in non-quasiequilibrium flow $\Pi$ and $\zeta$ fix the shear stress profiles.

From the above philosophy and conjectures it can be seen that for closure we need Eq. (41). This relationship would probably need to be deduced from experiment. Unfortunately, this relation is for infinite $S$ and experiments are carried out at finite $S$. Perhaps some sort of extrapolation scheme could be devised for obtaining (41). Whatever is devised it is obvious that a great deal of high quality experiments will be required. Large gaps will exist in the data no matter how extensive or thorough the experimental program is, and some sort of modeling might be needed to curve fit the results for the purposes of extrapolation and interpolation. As in the past, the intention of turbulence modeling is to give rational tools for curve fitting.

A promising candidate for such modeling is the attached eddy hypothesis.

\section{APPLICATION OF THE ATTACHED EDDY HYPOTHESIS}

Figure 15 shows schematically a representative attached eddy in a turbulent boundary layer. Work by Grass et $a l .^{23}$ and Smith $e t a .^{24}$ shows that hairpin or horseshoe or $\cap$-shaped eddies form in wall turbulence but undergo a whole variety of distortions (see also Robinson ${ }^{25}$ ). However, the authors are using the term "representative eddy" in the Townsend sense. This is a statistical eddy which is the simplest shape that will give the desired link between the mean flow and Reynolds shear stress distribution. This eddy leans in the streamwise direction at a fixed angle, its height is $\delta$ and it can be seen to contribute to a spanwise component of vorticity. If there is a random array of such

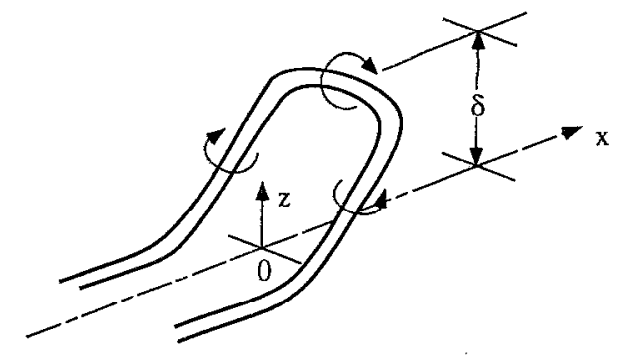

FIG. 15. Sketch of attached eddy of length scale $\delta$.

eddies with an average surface density proportional to $1 / \delta^{2}$, then the contribution from such an array of height $\delta$ to the mean vorticity is given by $\xi$, where

$$
\xi=\frac{U_{\tau}}{\delta} f\left[\frac{z}{\delta}\right]
$$

where $U_{\tau}$ is the characteristic velocity scale of the eddy (assumed to be the friction velocity). The function $f$ depends on eddy shape and can be found by finding the mean spanwise component of vorticity on horizontal sectioning planes (see Perry and Chong ${ }^{26}$ and Perry et al.). ${ }^{2}$ Further details may be found in Perry ${ }^{27}$ and Perry et al. ${ }^{28}$ From the Biot-Savart law the contribution to the Reynolds stress $\Delta\left(u_{i} u_{j}\right)$ is given by

$$
\frac{\Delta\left(\bar{u}_{i} u_{j}\right)}{U_{\tau}^{2}}=I_{i j}\left[\frac{z}{\delta}\right]
$$

Here $u_{i}$ are fluctuating components of velocity, i.e. $u_{1}=u^{\prime}, u_{2}=v^{\prime}$, and $u_{3}=w^{\prime}$ used carlier. This application of the Biot-Savart law need be applied to only one eddy and an image of it in the wall. Again this is outlined in Perry et $a .^{2}$ Introducing logarithmic variables

$$
\lambda=\ln \left[\frac{\delta}{z}\right], \quad \lambda_{1}=\ln \left[\frac{\delta_{1}}{z}\right], \quad \lambda_{E}=\ln \left[\frac{\delta_{c}}{z}\right]
$$

it can then be shown with an array of eddies randomly distributed over the wall that the gradient of the mean velocity defect $U_{D}^{*}=\left(U_{1}-U\right) / U_{\tau}$ and the Reynolds stress $\overline{u_{i} u_{j}}$ are given by two convolution integrals

$$
\begin{aligned}
& \frac{d U_{D}^{*}}{d \lambda_{E}}=\int_{-\infty}^{\infty} M h[\lambda] e^{-\lambda} \omega\left[\lambda-\lambda_{E}\right] T\left[\lambda-\lambda_{E}\right] d \lambda, \\
& \frac{\overline{u_{i} u_{j}}}{U_{\tau}^{2}}=\int_{-\infty}^{\infty} M J_{i j}[\lambda] \omega\left[\lambda-\lambda_{E}\right] T^{2}\left[\lambda-\lambda_{E}\right] d \lambda
\end{aligned}
$$

where eddies have scales $\delta$ ranging from $\delta_{1}$ (the scale of the smallest eddy $\approx 100 v / U_{\tau}$ ) to $\delta_{c}$, the scale of the largest eddy which is equal to the boundary layer thickness. It is assumed that all eddies are geometrically similar. In (46) and (47) $h[\lambda]=f[z / \delta]$ and $J_{i j}[\lambda]=I_{i j}[z / \delta]$. (In some earlier publications, ${ }^{3} h[\lambda]$ was written as $f[\lambda]$ etc. The present notation is more correct.)

Equations (46) and (47) were first derived by Perry, $\mathbf{L i}$, and Marusicic ${ }^{3}$ and are a generalization of Townsend's ${ }^{1}$ attached eddy hypothesis. However, they incorporated a change of eddy shape with scale which the authors pres- 

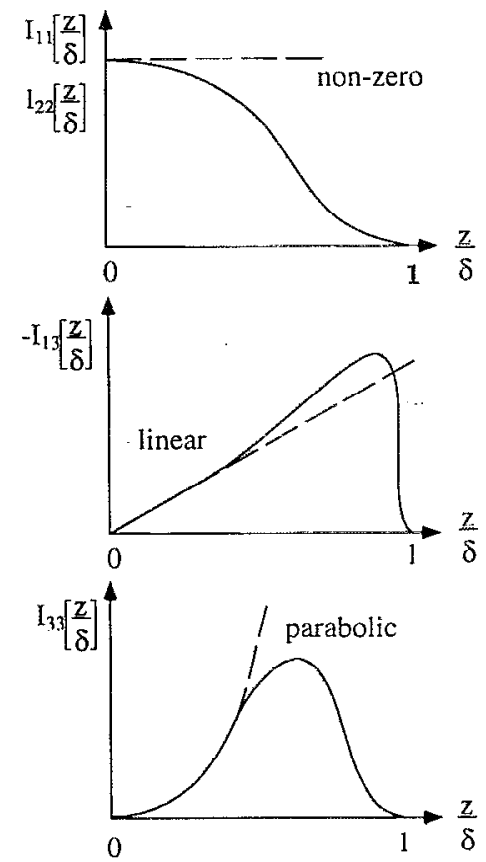

FIG. 16. Townsend's eddy intensity functions. Sketch only.

ently consider to be unnecessary at this stage. Here $\omega$ is a weighting function which is a measure of how the PDF of eddy scales differ from an inverse power law in $\delta ; T$ is a weighting function which is a measure of how the velocity scale of eddies of a given scale $\delta$ differ from $U_{\tau}$. The function $\omega$ switches to zero when $\lambda<\lambda_{1}$ and $\lambda>\lambda_{E}$. This effectively controls the limits of the integration.

The constant $M$ in (46) and (47) is universal and its value depends on how $f$ and $I_{i j}$ are normalized. For $\delta / \delta_{c} \rightarrow 0$ but $\delta>\delta_{1}, \omega$ and $T$ are unity and this ensures that we obtain the logarithmic defect law and $-\overline{u_{1} u_{3}} / U_{\tau}^{2} \rightarrow 1$ for " $z / \delta_{c}$ sufficiently small. It is assumed that $\delta_{c} / \delta_{1}>1$, where this is actually proportional to the von Kármán number. The functions $I_{i j}[z / \delta]$ are the Townsend eddy intensity functions and sketches are shown in Fig. 16. Of particular importance is the behavior of $I_{i j}[z / \delta]$ as $z / \delta \rightarrow 0$. This behavior can be derived from Townsend's inviscid boundary condition applied at $z / \delta=0$. These eddies are assumed to ride over the boundary with slip. For simplicity we will assume that a representative eddy is a " $\Pi$-shaped" eddy, as shown in Fig. 17. This gives a Dirac delta function for $h[\lambda]$ and $I_{13}[z / \delta]$ will be assumed to be a triangle. This is not precisely the case but is close. It satisfies the Townsend ${ }^{1}$ inviscid boundary condition and also shows the rapid drop in the far field influence of the eddy for $z>\delta$. The triangle distribution and the delta function are very convenient functions for convolution integrals and although they are approximations they are adequate for demonstration purposes here. Suppose we made $\omega$ and $T$ equal to unity for all $\lambda-\lambda_{E}$ i.e., all $\delta / \delta_{c}$. Equation (46) will give a pure logarithmic defect profile and we know

$$
\frac{d U_{D}^{*}}{d \lambda_{E}}=\frac{1}{\kappa}
$$
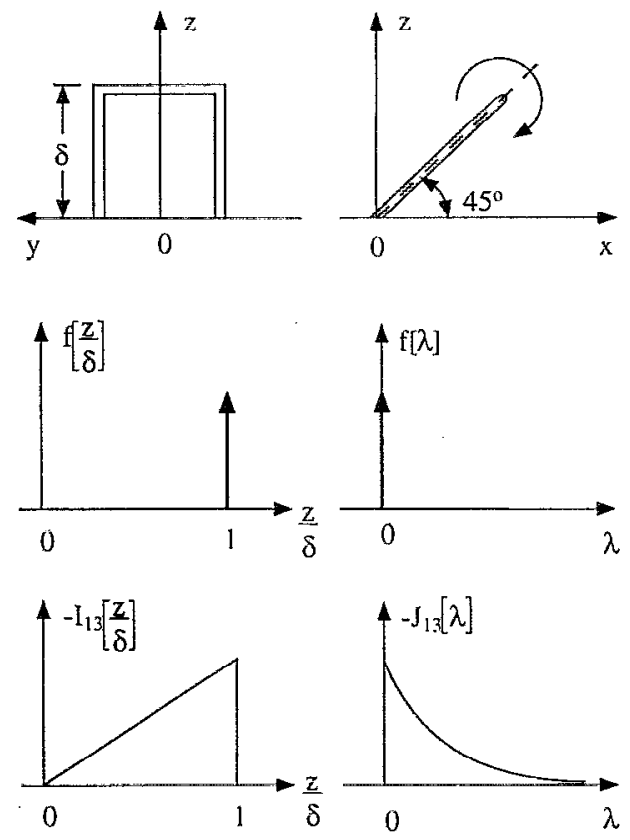

FIG. 17. "II-shaped" eddy details, its vorticity distribution, and the assumed triangular eddy intensity distribution.

and this sets the value of $M$ and thus the normalizing factor for $h[\lambda] e^{-\lambda}$ is known. Thus $M f[\lambda] e^{-\lambda}$ is known. Equation (47) gives a linear stress distribution approaching 1 for $z / \delta_{c} \rightarrow 0$. This boundary condition sets the normalization for $M J_{13}[\lambda]$. This case could represent flow in a duct with parallel walls since we have a logarithmic law in velocity defect from the wall to the center and $\tau / \tau_{0}$ is linear going from 1 at $z / \delta_{c}=0$ to 0 at $z / \delta_{c}=1$. This result is completely at variance with standard eddy viscosity or mixing length theories. These would insist on a constant shear stress for a logarithmic profile of mean velocity whereas here we have a linear stress. Other mean velocity and shear stress profiles can be correctly represented with this formulation by appropriate and plausible variations of $T$ and $\omega$ with $\left(\delta / \delta_{c}\right)$. The formulation fits in very naturally with the logarithmic law of the wall and law of the wake model and shear stress profiles of the correct shape can be easily generated.

Let us see if we can derive a function $\beta_{a}=\beta_{a}[\Pi]$ for quasiequilibrium flow with simple hypotheses. We will use the zero pressure gradient layer as a basic flow and attempt to use only empirical constants belonging to this zero pressure gradient case. For this example we will take $\Pi=0.55$.

In Eq. (46) $d U_{D}^{*} / d \lambda_{E}$ is known since $\Pi$ is known; $M h[\lambda] e^{-\lambda}$ is known as was determined above since we are assuming a " $\Pi$ eddy" shape. If we deconvolve (46) we obtain $\omega T$. Now from the momentum theory for zero pressure gradient it can be shown from (8) that for $\beta=0$ and $\delta_{c} d \Pi / d x=0$

$$
\lim _{S \rightarrow \infty} \frac{\tau}{\tau_{0}}=1+\frac{\eta f-\int_{0}^{\eta} f d \eta}{C_{1}}
$$

This has the interesting property that 


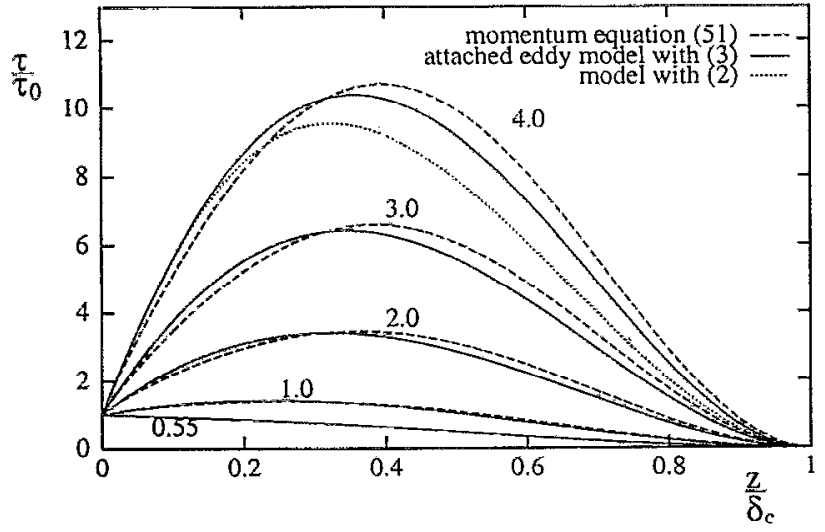

FIG. 18. Comparison of the attached eddy model shear stress with the momentum equation (51) result.

$$
\lim _{s \rightarrow \infty} \frac{\partial \tau / \tau_{0}}{\partial \eta}=-\frac{1}{\kappa C_{1}}
$$

Here again we see, this time from momentum, that a logarithmic profile in mean velocity can be consistent with a linearly varying stress distribution. Tennekes and Lumley ${ }^{29}$ (pp.192-193) reach the same conclusion. Hence the LHS of (47) is known; $M J_{13}[\lambda]$ is known and by a deconvolution $\omega T^{2}$ is known. From this and the previous result, $\omega$ and $T$ are known separately for the zero pressure gradient case.

Let it now be assumed that

$$
\omega=\omega\left[\lambda-\lambda_{E}\right]
$$

and

$$
T=T\left[\lambda-\lambda_{E}, \Pi\right],
$$

i.e. $\omega\left[\lambda-\lambda_{E}\right]$ is universal for equilibrium and quasiequilibrium flow but $T$, the weighting factor for velocity scales, depends on $\Pi$ as well as $\lambda-\lambda_{E}$. It has been found from cursory studies that this set of assumptions lead to the largest maximum shear stress in adverse pressure gradient layers and this is consistent with equilibrium layers as mentioned in Sec. II. Let us now apply (46) and (47) to pressure gradient flows. From (46) if we know $\Pi$ we know the LHS. We know $M h[\lambda] e^{-\lambda}$ since eddy shape will be assumed to be independent of pressure gradient. Now, $\omega\left[\lambda-\lambda_{E}\right]$ is known since it was obtained from the zero pressure gradient case and is taken to be universal. By a deconvolution of (46) we obtain $T\left[\lambda-\lambda_{E}, \mathrm{II}\right]$. We now substitute this into Eq. (47). We know $M J_{13}[\lambda]$ and $\omega\left[\lambda-\lambda_{E}\right]$ since it is universal and finally from a convolution of (47) we determine $-\overline{u_{1} u_{3}} / U_{\tau}^{2}$.

Now it is known from (8) that for $\delta_{c} d \Pi / d x=0$

$$
\lim _{S \rightarrow \infty} \frac{\tau}{\tau_{0}}=1+\frac{2 \beta_{a} \eta f}{C_{1}}+\frac{\eta f-\int_{0}^{\eta} f d \eta}{C_{1}} .
$$

By varying $\beta_{a}$ we match (51) to the convolution integral result obtained from (47) above thus establishing a relationship between $\beta_{a}$ and $\Pi$. In Fig. 18 the matched $\tau / \tau_{0}$ distributions given by (51) and (47) are shown. The
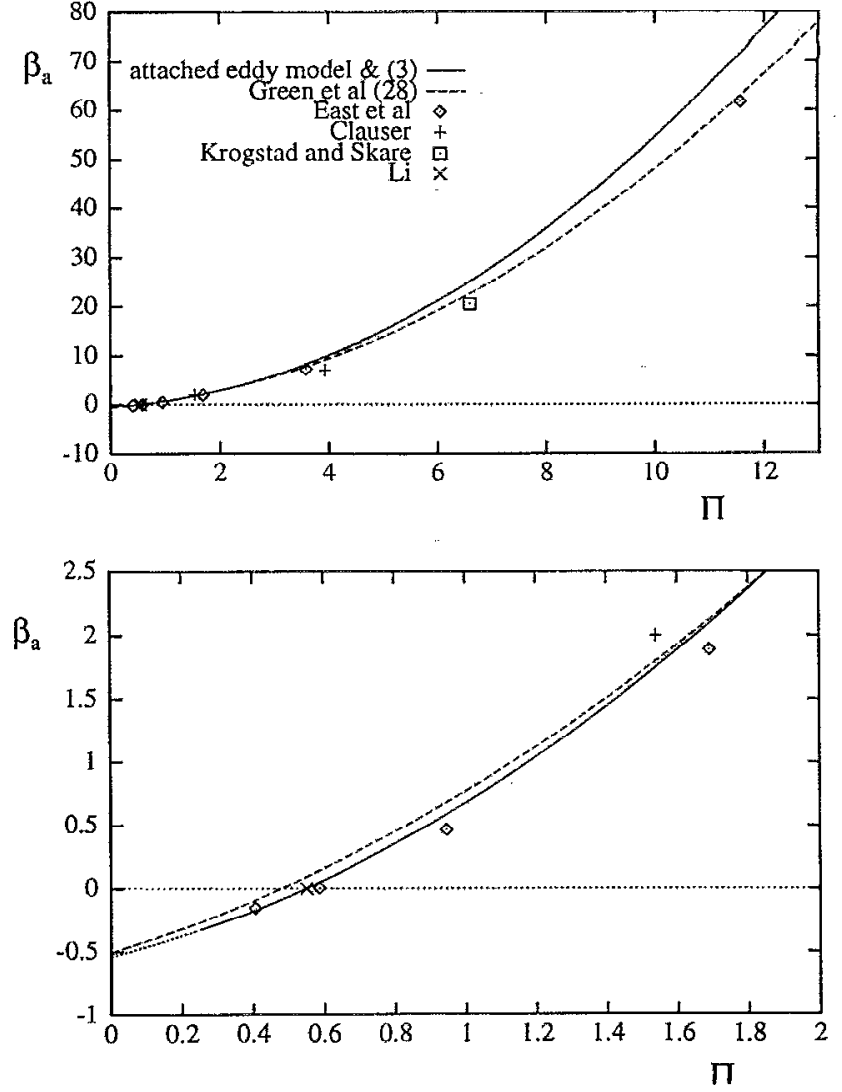

FIG. 19. Attached eddy model prediction compared to empirical curve-fit of Green et al. ${ }^{18}$ and experimental data of East et al. ${ }^{17}$ Clauser, ${ }^{6} \mathrm{Krogs-}$ tad and Skare ${ }^{32}$ and $\mathrm{Li}^{9}{ }^{9}$ For the model formulation (3) is used for $\Pi>0.25$ (solid line) and formulation (2) is used for $\Pi<0.25$ (dotted line).

matching was based on a least squares error criterion. For the particular arbitrary choices made for $f$ and $I_{13}$, it is seen that the trigonometric Coles wake function given by (3) works better than the Lewkowicz formulation (2) as seen for the $I I=4$ case. On the other hand, the results from (51) and (28) are insensitive to the choice between these two wake functions. Figures $19(a)$ and $19(b)$ show $\beta_{a}$ versus $\Pi$. Equation (3) was used for $\Pi>0.25$ but (2) was used for $\Pi<0.25$ because of problems with $\gamma$ mentioned in Sec. II. Considering the present crudity of the model, the results look promising.

Once the correct eddy shape is known the attached eddy hypothesis should give the broadband distributions of $\overline{u_{1}^{2}} / U_{\tau}^{2}, \overline{u_{2}^{2}} / U_{\tau}^{2}, \overline{u_{3}^{2}} / U_{\tau}^{2}, \bar{u}_{1} u_{3} / U_{\tau}^{2}$, and all spectral distributions (see Perry et al. ${ }^{2}$ and Perry and $\mathrm{Li}^{11}$ ). However, additional contributions from the Kolmogorov subrange would also need to be incorporated by further eddy structures additional to the attached eddies.

For the case of non-quasiequilibrium flow both $\omega$ and $T$ will probably be functions of both $\lambda-\lambda_{E}$ and $\Pi$. It is hoped that with the aid of experiment and hypotheses, some further valid physical insights into eddy structure will emerge. 


\section{CONCLUSIONS AND DISCUSSION}

It appears that with the logarithmic law of the wall and the law of the wake formulations together with the selfpreserving flow hypothesis of Rotta, ${ }^{4}$ that approximate equilibrium and quasiequilibrium layers have their nondimensional shear stress distributions completely specified once $\Pi$ is specified. Thus $\Pi$ specifies completely both the nondimensional velocity defect distribution and shear stress distribution. This is equivalent to having a universal distribution of eddy viscosity given by

$$
\frac{\epsilon}{\delta_{c} U_{\tau}}=\phi[\eta, \mathrm{II}]
$$

Such a relationship was first anticipated by Clauser $^{7}$ and later developed in closely related closure schemes by $\mathrm{Ce}-$ beci and Smith $^{30}$ and Mellor and Gibson. ${ }^{20}$ Thus equilibrium and quasiequilibrium conditions imply that Eq. (52) should be valid. This of course does not necessarily mean that a gradient diffusion mechanism is at work, but it is simply that by default equations of the form of (52) work here. Such equations always work if there is a simultaneous self similarity in velocity defect and shear stress (e.g. plain mixing layers and under some conditions jets and wakes). As soon as $\xi\left(=S \delta_{c} d \Pi / d x\right)$ has any effect (as is the case in the Marušić layer) Eq. (52) would come to grief if used as a closure hypothesis in a standard differential field method. On the other hand, one could say that the method developed here for equilibrium and quasiequilibrium flow is effectively an integral version of a Cebeci and Smith type of method but it has built in a warning which indicates when (52) is breaking down. Furthermore, closure does not come via an equation like (52) but an equation which gives the asymptotic value of $\beta_{a}$ in terms of $\Pi$, i.e.

$$
\beta_{a}=\beta_{a}[\mathrm{II}] \text {. }
$$

This may be obtained experimentally perhaps if we know how to extrapolate to infinite $S$ or with the aid of something like the attached eddy hypothesis. Such a hypothesis with its convolution integrals used in conjunction with the integral scheme proposed here is consistent with Townsend's ${ }^{31}$ statements about modcling of wall turbulence. Rather than using exchange coefficients related to local flow variables, the layers should be looked at as an "integrated whole" with the transport properties at one point being related to motions in regions remote from the point of interest.

For the more general class of layers the influence of $S \delta_{c} d \Pi / d x=\xi$ is appreciable and it is conjectured that we need a relation like

$$
\beta_{a}=\beta_{a}[\Pi, \zeta] \text {. }
$$

The functional form of this could be deduced from systematic experimental data and the attached eddy hypothesis could be a useful curve-fitting device for interpolation and extrapolation. Perhaps by monitoring the behavior of the weighting functions $\omega$ and $T$ in experiments, some clues for further hypotheses may emerge.

Of course, everything treated here depends on the Rotta ${ }^{4}$ hypothesis, that is, if conditions are found from the mean continuity and momentum equations for giving self similarity, then if these conditions are applied self similarity will occur. This may not happen because even though the conditions are necessary they may not be sufficient. Further systematic experiments are needed to verify this hypothesis.

\section{ACKNOWLEDGMENTS}

The first author gratefully acknowledges the help resulting from many stimulating discussions with Dr. D. Coles and Dr. H. G. Hornung of G.A.L.C.I.T. The Fairchild Fellowship Scheme is gratefully acknowledged for enabling the first author to spend the calendar year 1992 at G.A.L.C.I.T. where he carried out much of this work. The authors acknowledge the Australian Research Council for the financial support of other aspects of this project being carried out in Australia.

\section{APPENDIX: SOME DETAILS OF ANALYSIS}

Substituting (4) and (5) into (6) and integrating we obtain

$$
\frac{\tau}{\tau_{0}}=1+A_{1} X_{1}+A_{2} X_{2}+A_{3} X_{3}+A_{4} X_{4}
$$

where

$$
A_{i}=A_{i}[\eta, \mathrm{II}, S]
$$

where

$$
\begin{aligned}
& X_{1}=\frac{d \delta_{c}}{d x}, \quad X_{2}=\frac{\delta_{c}}{S} \frac{d S}{d x}, \\
& X_{3}=\delta_{c} \frac{d \Pi}{d x}, \quad X_{4}=\frac{\delta_{c}}{U_{1}} \frac{d U_{1}}{d x} .
\end{aligned}
$$

The $A_{i}$ 's are given by

$$
\begin{array}{ll}
A_{1}=\psi_{3}+S \psi_{4}, & A_{2}=-\psi_{1}-S \psi_{2}, \\
A_{3}=\psi_{6}+S \psi_{7}, & A_{4}=S \psi_{5}+\psi_{1}+S \psi_{2},
\end{array}
$$

where

$$
\begin{aligned}
& \psi_{1}=2 e_{2}-e_{3}, \quad \psi_{2}=-e_{1}, \\
& \psi_{3}=e_{2}-e_{3}, \quad \psi_{4}=e_{4}-e_{1}, \\
& \psi_{5}=e_{4}-2 e_{1}, \quad \psi_{6}=e_{6}-e_{7}, \\
& \psi_{7}=-e_{5},
\end{aligned}
$$

and where

$$
\begin{aligned}
& e_{1}=\int_{0}^{\eta} f d \eta, \quad e_{2}=\int_{0}^{\eta} f^{2} d \eta \\
& e_{3}=f \int_{0}^{\eta} f d \eta, \quad e_{4}=\eta f \\
& e_{5}=\frac{d}{d \Pi} \int_{0}^{\eta} f d \eta, \quad e_{6}=\frac{d}{d \Pi} \int_{0}^{\eta} f^{2} d \eta, \\
& e_{7}=f \frac{d}{d \Pi} \int_{0}^{\eta} f d \eta .
\end{aligned}
$$


This is the form arrived at by Perry and $\mathrm{Li}^{11}$ and in an equivalent form by Marušić. ${ }^{10}$ Now relationships can be established between the $X_{i}$ 's as follows. From the logarithmic law of the wall and law of the wake

$$
X_{2}=E_{1}\left(X_{1}+N X_{3}+X_{4}\right)
$$

where

$$
E_{1}=\frac{1}{\kappa S+1}
$$

and

$$
N=W_{c}[1, \Pi]+\Pi \frac{d W_{c}[1, \Pi]}{d \Pi} .
$$

From the outer boundary condition $\tau / \tau_{0}=0$ at $\eta=1$

$$
0=1+B_{1} X_{1}+B_{2} X_{2}+B_{3} X_{3}+B_{4} X_{4}
$$

where

$$
B_{i}=A_{i}[1, \Pi, S] \text {. }
$$

From Eqs. (A2)-(A5) $X_{1}$ and $X_{2}$ can be expressed in terms of $X_{3}$ and $X_{4}$ to give

$$
\frac{\tau}{\tau_{0}}=f_{1}[\eta, \Pi, S]+f_{2}[\eta, \Pi, S] \delta_{c} \frac{d \Pi}{d x}+f_{3}[\eta, \Pi, S] \frac{\delta_{c}}{U_{1}} \frac{d U_{1}}{d x} .
$$

Further equations which are useful for deriving the evolution relationships (20), (21), and (22) are

$$
\begin{aligned}
\Phi= & \frac{d \ln U_{\tau}}{d \ln \delta_{c}}=\frac{1}{U_{\tau}} \frac{d U_{\tau}}{d x}\left(\frac{1}{\delta_{c}} \frac{d \delta_{c}}{d x}\right)^{-1} \\
= & -\left(\frac{\kappa \beta}{C_{1}}\left(\frac{d \delta_{c}}{d x}\right)^{-1}+1\right) /(\kappa S+1), \\
\frac{d \theta}{d x}= & \left\{\frac{d \delta_{c}}{d x}\left[S\left(C_{1} \kappa S^{2}-C_{2} \kappa S+C_{2}\right)\right]\right. \\
& \left.-\left[-C_{1} S+2 C_{2}\right] \frac{\beta}{C_{1}}\right\} /\left(S^{3}(\kappa S+1)\right), \\
\frac{d \theta}{d x}= & \frac{1}{S^{2}}+\left(3 S C_{1}-2 C_{2}\right) \frac{\beta}{S^{3} C_{1}}, \\
\frac{d \delta_{c}}{d x}= & \frac{(\kappa S+1)+\left(3 \kappa C_{1} S-2 \kappa C_{2}+2 C_{1}\right) \beta / C_{1}}{\left(C_{1} \kappa S^{2}-C_{2} \kappa S+C_{2}\right)},
\end{aligned}
$$

and

$$
\delta_{c} \frac{d S}{d x}=-\frac{\beta}{C_{1}}-S \Phi \frac{d \delta_{c}}{d x}=R[S, \beta, \Pi] .
$$

${ }^{1}$ A. A. Townsend, The Structure of Turbulent Shear Flow (Cambridge University Press, Cambridge, 1976).

${ }^{2}$ A. E. Perry, S. M. Henbest, and M. S. Chong, "A theoretical and experimental study of wall turbulence," J. Fluid Mech. 165, 163 (1986).

${ }^{3}$ A. E. Perry, J. D. Li, and I. Marusić, "Towards a closure scheme for turbulent boundary layers using the attached eddy hypothesis," Philos. Trans. R. Soc. London Ser. A 336, 67 (1991).

4J. C. Rotta, "Turbulent boundary layers in incompressible flow," Prog. Aeronaut. Sci. 2, 1 (1962).

${ }^{5} \mathrm{C}$. M. Millikan, "A critical discussion of turbulent flows in channels and circular tubes," in Proceedings of the 5th International Congress on Applied Mechanics (Wiley, New York, 1938).

${ }^{6} \mathrm{~F}$. H. Clauser, "Turbulent boundary layers in adverse pressure gradients," J. Aeronaut. Sci. 21, 91 (1954).

${ }^{7}$ F. H. Clauser, "The turbulent boundary layer," Adv. Mech. 4, 1 (1956).

${ }^{8}$ D. E. Coles, "Remarks on the equilibrium turbulent boundary layer," $J$. Aeronaut. Sci. 24, 459 (1957).

${ }^{9} \mathrm{~J}$. D. Li, "The turbulence structure of wall shear flow," Ph.D. thesis, University of Melbourne, 1989.

${ }^{10} \mathrm{I}$. Marušić, "The structure of zero- and adverse-pressure gradient turbulent boundary layers," Ph.D. thesis, University of Melbourne, 1991.

${ }^{11}$ A. E. Perry and J. D. Li, "Theoretical and experimental studies of shear stress profiles in two dimensional turbulent boundary layers," Report No. FM-18, Department of Mechanical Engineering, University of Melbourne, 1991.

${ }^{12}$ A. E. Perry, "A theoretical study of equilibrium turbulent boundary layers" (originally unpublished, 1968), Report No. FM-19, Department of Mechanical Engineering, University of Melbourne, 1992.

${ }^{13}$ A. E. Perry, "A new look at some closure problems of turbulent boundary layers," GALCIT Report No. FM-92-4, California Institute of Technology, 1992.

${ }^{14}$ D. E. Coles, "The law of the wake in the turbulent boundary layer," J. Fluid Mech. 1, 191 (1956).

${ }^{15}$ A. K. Lewkowicz, "An improved universal wake function for turbulent boundary layers and some of its consequences," $Z$. Flugwiss. Weltraumforsch. 6, 261 (1982).

${ }^{16} \mathrm{~A}$. E. Perry and J. D. Li, "Experimental support for the attached eddy hypothesis in zero-pressure-gradient turbulent boundary layers," $\mathrm{J}$. Fluid Mech. 218, 405 (1990).

${ }^{17}$ L. F. East, W. G. Sawyer, and C. R. Nash, "An investigation of the structure of equilibrium turbulent boundary layers," R.A.E. Technical Report 79040, 1979.

${ }^{18} \mathrm{~J}$. E. Green, D. J. Weeks, and J. W. F. Brooman, "Prediction of turbulent boundary layers and wakes in compressible flow by a lagentrainment method," Aeronautical Research Council, Report and Memorandum (U.K.) 3791, 1973.

${ }^{19} \mathrm{~W}$. P. Jones and B. E. Launder, "Some properties of sink flow turbulent boundary layers," J. Fluid Mech. 56, 337 (1972).

${ }^{20}$ G. L. Mellor and D. M. Gibson, "Equilibrium turbulent boundary layers," J. Fluid Mech. 24, 225 (1966).

${ }^{21}$ F. M. White, Viscous Fluid Flow (McGraw-Hill, New York, 1974).

${ }^{22}$ D. E. Coles, "The turbulent boundary layer in a compressible fuid," USAF The Rand Cooperation, Report No. R-403-PR, Appendix A, 1962. See also Phys. Fluids 7, 1403 (1964).

${ }^{23}$ A. J. Grass, K. J. Stuart, and M. Mansour-Tehrani, "Vortical structures and coherent motion in turbulent flow over smooth and rough boundaries," Philos. Trans. R. Soc. London Ser. A 336, 35 (1991).

${ }^{24}$ C. R. Smith, J. D. A. Walker, A. H. Haidari, and U. Sobrun, "On the dynamics of near-wall turbulence," Philos. Trans. R. Soc. London Ser. A 336, 67 (1991).

${ }^{25}$ S. K. Robinson, "Coherent motions in the turbulent boundary layer," Annu. Rev. Fluid Mech. 23, 601 (1991).

${ }^{26}$ A. E. Perry and M. S. Chong, "On the mechanism of wall turbulence," J. Fluid Mech. 119, 173 (1982).

${ }^{27}$ A. E. Perry, "Turbulence modelling using coherent structures in wakes, plane mixing layers and wall turbulence," in Perspectives in Turbulence Studies, edited by H. U. Meier and P. Bradshaw (Springer-Verlag, Berlin, 1987), pp. 115-153.

${ }^{28}$ A. E. Perry, J. D. Li, S. M. Henbest, and I. Marưić, "Attached eddy hypothesis in wall turbulence," in Near Wall Turbulence, edited by S. J. Kline and N. H. Afgan (Hemisphere, New York, 1990), pp. 715-735.

${ }^{29} \mathrm{H}$. Tennekes and J. L. Lumley, A First Course in Turbulence (MIT Press, Cambridge, MA, 1972).

${ }^{30}$ T. Cebeci and A. M. O. Smith, Analysis of Turbulent Boundary Layers (Academic, New York, 1974).

${ }^{3}$ A. A. Townsend, "Equilibrium layers and wall turbulence," J. Fluid Mech. 11, 97 (1961).

${ }^{32}$ P.-A. Krogstad and P. E. Skåre, "An experimental investigation of a turbulent equilibrium boundary layer near separation," in Near-Wall Turbulent Flows, edited by R. M. C. So, C. G. Speziale, and B. E. Launder (Elsevier Science, Amsterdam, 1993), pp. 911-920. 


\section{University Library}

\section{- M M N E R VA A gateway to Melbourne's research publications}

Minerva Access is the Institutional Repository of The University of Melbourne

Author/s:

Perry, A. E.;Marusic, I.;Li, J. D.

Title:

Wall turbulence closure based on classical similarity laws and the attached eddy hypothesis

Date:

1994

Citation:

Perry, A. E., Marusic, I. \& Li, J. D. (1994). Wall turbulence closure based on classical similarity laws and the attached eddy hypothesis. American Institute of Physics, 6(2), 1024-1035.

Publication Status:

Published

Persistent Link:

http://hdl.handle.net/11343/34806 